\section{Setting radiation standards}

SIR-The setting of standards of protection against ionizing radiation, already controversial, is being confused by the difficulty of specifying clearly the significance of the risk to an exposed individual. ' $R$ isk' is used here to mean the probability of a deleterious event. The term 'detriment' can be used to combine the probability and the severity of that event.

For many years, the fatal risk to an individual has been presented as the probability of attributable death with no allowance for competing causes of death. The effect of this allowance is known, but has not been used explicitly in setting standards. The effect of latency on the degree of detriment has also been neglected, although a death in later years is less detrimental than an earlier one.

More recently, in its 1988 report to the UN General Assembly', the United Nations Scientific Committee on the Effects of Atomic Radiations (UNSCEAR) has addressed both these issues. The allowance for competing causes of death is explicitly included in the risk estimates, and the effect of latency on the detriment is indicated by estimating the average years of life lost. Information on the distribution of risk with time after exposure is still incomplete because many members of the exposed populations being studied are still alive. UNSCEAR offers two risk-projection models, one with the risk constant (after a latent period) - an additive model - and the other with the risk increasing with time as does the risk of 'natural' cancer - a multiplicative model. The letter predicts about twice as many deaths as the former

\title{
$\mathrm{X}$-chromosome reactivation and ageing
}

SIR--At first sight it seems that the results of Migeon et al. ${ }^{\prime}$ on the low reactivation frequency of the human X-linked hypoxanthine phosphoribosyl transferase (HPRT) gene do not confirm the earlier finding of the strong age-related reactivation of the mouse $\mathrm{X}$-linked ornithine carbamoyl transferase gene (OCT) ${ }^{2}$. Whereas the spontaneous reactivation of the silent OCT gene was increased 50-fold in mice older than one year, the reactivation of the silent human HPRT gene in female Lesch-Nyhan heterozygotes is rare at any age.

In studies of the process of ageing, one of the main challenges is to understand how similar physiological or biochemical events occur at very different rates in species with different lifespans. For example the crosslinking of collagen is known to increase with age, but although the chemistry is the same, the rates of reaction are very different in different species ${ }^{3}$. The same is true for the accum- with no more years of lost life. Unfortunately, most commentators on this topic without considering the decrease in years lost per attributable death.

There is a further weakness in using risk alone. The total risk of death cannot be increased; it is unity. Radiation can change the time and cause of death, not the overall probability.

The risk to the individual is thus a poor measure of the consequences of exposure to ionizing radiation. We need a multiattribute approach to take account of the time and nature of a death in addition to its probability. This would also make it easier to include explicitly the non-fatal effects such as curable cancer and hereditary defects. It is to be hoped that those reponsible for setting standards, particularly dose limits, will not rush into changes based only on the simple concept of risk. As both the International Commission on Radiological Protection ${ }^{2}$ and the National Radiological Protection Board ${ }^{3}$ have indicated, the most appropriate immediate reaction is to increase the attention paid to the need to keep all exposures as low as can reasonably be achieved. Quantitative changes in standards should be a more measured response.

\section{Thames Street, St Ebbes,}

\section{Oxford $O X I I S U, U K$}

United Nations Scientific Committee on the Effects of Atomic Radiation. Sources, Effects and Risks of lonizing Radiation (United Nations. New York, I988)

Stutement from the 1987 Como meeting of the International Commission on Radiological Protection Radiological Protection Bull. Suppl. 86 (1987)

Protection Bull. Suppl. 86 (1987).
Clarke, R.H. NRPB (Guidance on Risk Estimates and Dose Limits, Radiological Protection Bull. 88 (1988). have stressed the increase in fatal risk

DNA in primary diploid cells is much more effective in human than in mouse cells, and that hamster cells have an intermediate level of maintenance 5 .

Two of the results reported by Migeon et al. together indicate that there may be a low loss of methylation in normal human cells with ageing.

First, there is a slight increase with age in the proportion of cells with active HPRT, which is an insensitive assay, and the results are of marginal statistical significance $(P=0.08)$.

Second, using a much more sensitive assay, it is shown that the silent HPRT gene can be more easily reactivated by the demethylating agent deoxyazacytidine in cells from old individuals than in cells from young ones $(P<0.005)$.

These results would be expected if the methylation of many sites in the $\mathrm{CpG}$-rich island of the human HPRT gene declines slowly with age, but the overall silencing mechanism is fairly efficiently maintained. However, a gradual loss of methylation would increase the probability of reactivation by a demethylating agent. If this interpretation is correct, then similar experiments carried out with female 'Lesch-Nyhan' heterozygous mice ${ }^{6.7}$, should show a significant age-related reactivation of HPRT. Such a result would reinforce the view that cellular homoeostasis is much better maintained in longlived species than in short-lived ones ${ }^{4.8}$.

RoBin Holliday

CSIRO Laboratory for Molecular Biology,

PO Box 184, North Ryde,

New South Wales 2113, Australia

Migcon B.R., Axelman, J. \& Beggs, A.H. Nature 335 93-96 (1988).

Wareham, K.A. Lyon, M.F., Glenister. P.H. \& Williams, E.D. Nature 327. 725-727 (1987)

Yamauchi, M Woodley, D.T \& Mechanic, G.L. Biochem biophys, Res. Commun. 152, $898-903$ (1988)

Biochem. hiophys. Res. Commun. 152, $898-9.170$
Holliday, R. Science 258, 163-170 (1987).

Holliday, R. Science 258, 163-170) (1987).
Wilson, V.L. \& Jones, P. A. Science 220, 1055-1057 (1983)

Wilson, V.L. \& Jones, P.A. Science 220, 1055-1057(1983)
Hooper, M. Hardy, K., Handyside, A.. Hunter. S. \& Monk, M. Nature 326, 292-295 (1987)

Kuehn, M.R.. Bradley, A., Robertson E.J. \& Evans, M.J. Nature 326. 295-298 (1987).

Rolliday, R Perspect hiol Med 32, 109-123 (1988).

denaturation of lens crystallin and other 'biomarkers' of ageing, as well as many pathologies, including the appearance of neoplasms. It is well known that mouse cells transform to malignant cells readily in culture, whereas human cells are very refractory to this transformation. This makes biological sense, because on a per cell basis, small short-lived animals have a far higher incidence of cancer than large long-lived ones.

It is therefore reasonable to argue that the age-related reactivation of a silent $\mathrm{X}$ linked gene would be many times higher in mouse cells than in human cells. Another way of saying the same thing is that the controls of cellular metabolism, or cellular homoeostasis, must be very much tighter in long-lived animals than in short-lived ones, and this would of course include the silencing of gene expression by DNA methylation ${ }^{+}$. It is already known that the maintenance of cytosine methylation in

\section{Misidentified cell}

SIR-Because the cell line TE671 is said to originate from an early culture of a human medulloblastoma', it has been widely used to investigate this tumour type and as a model for primitive neural cells in general. And because the cell line can be infected by the AIDS virus $\mathrm{HIV}^{2}$ (in a way that cannot be blocked by soluble CD4, as reported on page 368 of this issue ${ }^{3}$ ), TE671 could provide an ostensibly useful in vitro model of HIV infection of the brain. Our evidence, however, suggests that TE671 is not a neural cell but a muscle cell.

We note that TE671 cells have a number of features in common with the rhabdomyosarcoma cell line RD. They have several of the phenotypic characteristics of striated muscle cells, including the presence of desmin, myoglobin ${ }^{4}$ and the 